\title{
Investigations on the ELM cycle by local 3D perturbation experiments
}

\author{
P.T. Lang, K. Gal ${ }^{o}$, J. Hobirk, S. Kalvin ${ }^{o}$, G. Kocsis ${ }^{o}$, V. Mertens, J. Neuhauser, \\ M. Maraschek, W. Suttrop, G. Veres ${ }^{o}$, ASDEX Upgrade Team \\ e-mail address of first author: peter.lang@ipp.mpg.de \\ Max-Planck-Institut für Plasmaphysik, EURATOM Association, \\ Boltzmannstr. 2, D-85748 Garching, Germany \\ ${ }^{o}$ KFKI-RMKI, EURATOM Association, P.O. Box 49, H-1525 Budapest-114, Hungary
}

\begin{abstract}
An experimental study is performed to investigate the underlying physics of ELM triggering by imposing local perturbations at the plasma edge. Deuterium is injected during type-I ELMy H-mode phases by solid small pellets or as a supersonic gas jet. In both cases the repetition rate is small compared to the intrinsic ELM frequency, aiming for a small perturbation of the intrinsic ELM cycle. Active triggering of ELMs requires a density perturbation in the gradient region about $1 \mathrm{~cm}$ inside the separatrix (measured at horizontal mid plane), a condition that is achieved by pellets of any size and speed but not even by the strongest available gas jets. The pellet-induced triggering of ELMs occurs always when pellets reach the H-mode barrier region. A density perturbation produced by a gas jet remains localized at the separatrix, and even if larger by a factor of at least 100, it is not sufficient to trigger an ELM. No significant dependence was found of the ELM size on the ELM onset time, measured from the onset of the previous ELM.
\end{abstract}




\section{Introduction}

In the ITER type-I ELMy H-mode standard operational scenario intrinsic ELMs can potentially lead to excessive power load to the divertor. ELM mitigation by forcing ELMs to occur at higher frequencies and consequently, lower energy loss of each individual ELM is considered a possible solution. Several ELM pace-making (or "pacing") techniques have been demonstrated, most successfully the injection of cryogenic hydrogen pellets [1] and the edge current induction by fast vertical plasma motion [2]. Pellets induce a periodic localized perturbation and can establish ELM pacing in case of a sufficiently strong impact on the local plasma density. With growing machine and plasma size, the global parameter changes produced by such local perturbations become less pronounced, thus our findings obtained at the mid-size tokamak ASDEX Upgrade scale favorably towards ITER. Obviously, there is still potential for optimization if the precise local trigger conditions become known. Consequently, the main aim of our studies is to map out the spatial and temporal variation of the ELM trigger threshold. In this way an optimized ELM pacing tool can be built which decouples unwanted side effects like pellet fueling from the pure ELM trigger effect.

\section{Experimental approach}

The experiments described here have been performed in the ASDEX Upgrade tokamak using a lower single null (LSN) plasma configuration and stationary ELMy H-mode phases with low $(\sim 30-40 \mathrm{~Hz})$ ELM frequency and a duration of several seconds. Detailed description of the configuration and heating scenario as well as the pellet injection system can be found in [3]. Pellet launch from the magnetic high field side (HFS) at an angle of $72^{\circ}$ with the respect to the horizontal mid plane can take place in the velocity range from 240 to $1000 \mathrm{~m} / \mathrm{s}$ using masses from about 2 to $35 \times 10^{19} \mathrm{D}$-atoms. The pellet injector is able to deliver up to 120 pellets at repetition rates $f_{P e l}$ up to $80 \mathrm{~Hz}$. For local edge perturbations supersonic gas jet pulses were applied as well. The gas injector delivered molecular D jet at a speed of $1.8 \mathrm{~km} / \mathrm{s}$ and typically $2 \mathrm{~ms}$ duration, containing a particle amount of $1-5 \times 10^{19} D_{2}$. It was installed at the magnetic low field side (LFS) slightly above the horizontal mid plane, distances to the plasma separatrix were altered between 6 and $12 \mathrm{~cm}$. Due to the low repetition rate of $2 \mathrm{~Hz}$, only a trigger proof-of-principle experiment was feasible. Details of the gas injection system and results of the dedicated study using this system can be found in [4]. In both cases the ELM trigger frequency (typically $6 \mathrm{~Hz}$ with pellets, and $2 \mathrm{~Hz}$ with the gas jet) were deliberately chosen low compared to the intrinsic ELM frequency, in order to minimize the perturbation of the background plasma parameters. In particular, the time delay between successive injection events was large compared to the relevant confinement times. This is to ensure the perturbation induced by the trigger event has decayed before the next active trigger event and in the subsequent analysis unperturbed background plasma conditions can be assumed.

Because of the random timing between spontaneously occurring ELMs and injection events, different trigger events occur at different times in the ELM cycle. Subsequently, we perform our analysis as a function of $d t_{e l .}$, the time interval between the trigger event and the previous spontaneous ELM.

In a series of different discharges (fixed settings in one discharge), the injected deuterium mass was varied (by varying the gas jet pressure or pellet size) in order to cause some variation of the density perturbation strength. Different pellet velocities $\left(v_{P}=240 \ldots 1000 \mathrm{~m} / \mathrm{s}\right)$ were employed and as this parameter affects the ablation rate the 
strength of the local density perturbation is changed as well.

Since the pellet velocity is known, the time difference between the onset of the pellet ablation and the begin of the growth if the ELM instability can be used to determine the position at which the pellet triggers the ELM. The time evolution of the pellet ablation is monitored by the $D_{\alpha}$ radiation from the ablation cloud [5]. Using a fast framing camera system and by matching the modeled and measured $D_{\alpha}$ evolution the pellet position as a function of time is determined. When analyzing the trigger position, one has to allow a finite growth time or onset time $t^{o s}$, i.e. a time lag between the local trigger action of the pellet and the first actual observation of a magnetic perturbation in the Mirnov diagnostic. The pellet flight time and the onset time $t^{o s}$ can be separated by using pellets with different velocities if one assumes that only the flight time varies with pellet velocity and $t^{o s}$ remains constant. The precision of the seed location depends on the accuracy of the ELM onset time and the pellet position. Details of the detection systems and the analysis will be reported elsewhere [6]. The main facts are the following: The assumption of the pellet moving with constant initial velocity along a straight path is sufficiently well met for an accuracy of the pellet position of about $5 \mathrm{~mm}$ with respect to the plasma flux surfaces. The accuracy of the ELM onset time is about $10 \mu s$.

\section{Results}

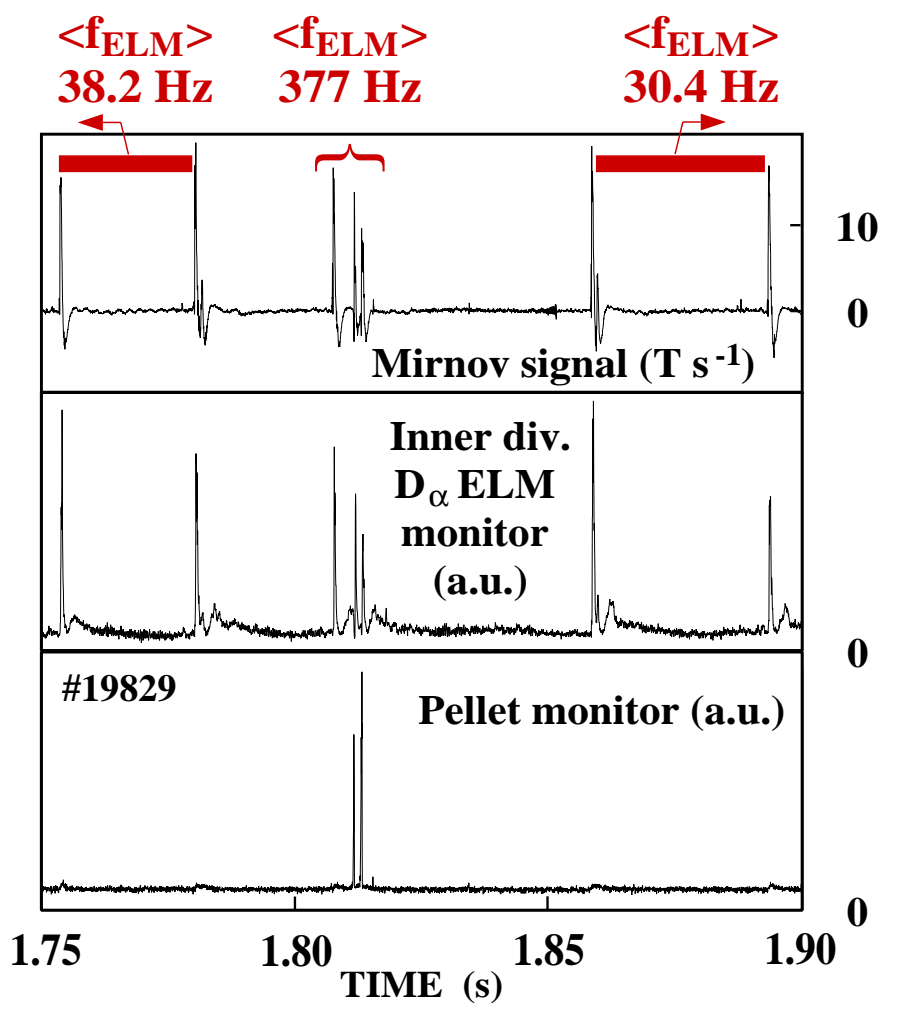

Figure 1: Two small pellet fragments penetrate within $1.4 \mathrm{~ms}$, shortly after the occurrence of a spontaneous ELM. Both pellet fragments trigger ELMs with a clear type-I signature.

So far we observe that every injected pellet triggers its own type-I ELM which occurs on a short time scale $\left(t^{o s}\right.$ of the order $100 \mu \mathrm{s}$ ) after the pellet crosses the plasma edge. In contrast to this, no immediate triggering of ELMs is 
observed with the supersonic gas jet, although it can alter the average duration of the ELM cycles (typically shorting it). This effect can be understood as a result of global particle fueling by the gas injection [4]. A main conclusion is that even very strong local density perturbations in the scrape off layer and the barrier region close to the separatrix are unable to trigger ELMs. To the contrary, even smallest deuterium pellets (mass $\sim 10^{18} \mathrm{D}$ atoms) are found to trigger ELMs. These pellets produce much smaller local particle deposition rates, even though the deposition region extends over the entire edge barrier region. This indicates that seed perturbations for ELM triggering must be imposed inside the $\mathrm{H}$-mode barrier region characterized by steep density and temperature gradients. In this region, moderate perturbations are sufficient to trigger ELMs while strong fueling in the vicinity of the separatrix is not; i.e. there is a pronounced spatial characteristics of the ELM trigger susceptibility.

Pellets can trigger ELMs in H-mode at any time. Obviously, the plasma edge is never stable against a pelletinduced seed perturbation. This can be seen from figure 1, which shows an ELM trigger event after a fragmented pellet arrives at the plasma edge soon after a spontaneous ELM. Two tiny pellet fractions penetrate within $1.4 \mathrm{~ms}$ into the plasma, the first one only $3.9 \mathrm{~ms}$ after the preceding spontaneous ELM. Both pellets cause individual ELM events with a clear type-I signature as can be seen from Mirnov and inner divertor $D_{\alpha}$ signals. An instantaneous ELM frequency can be defined as the reciprocal time difference between two ELMs, for example a pellet-induced ELM and the preceding ELM. In this case the instantaneous ELM frequency is increased to $377 \mathrm{~Hz}$ for the triple ELM sequence marked by the bracket, while the average ELM frequency is 38.2 and $30.4 \mathrm{~Hz}$ before and after this event, respectively. The ELM frequency can temporarily exceed by far the average frequency of spontaneous ELMs.

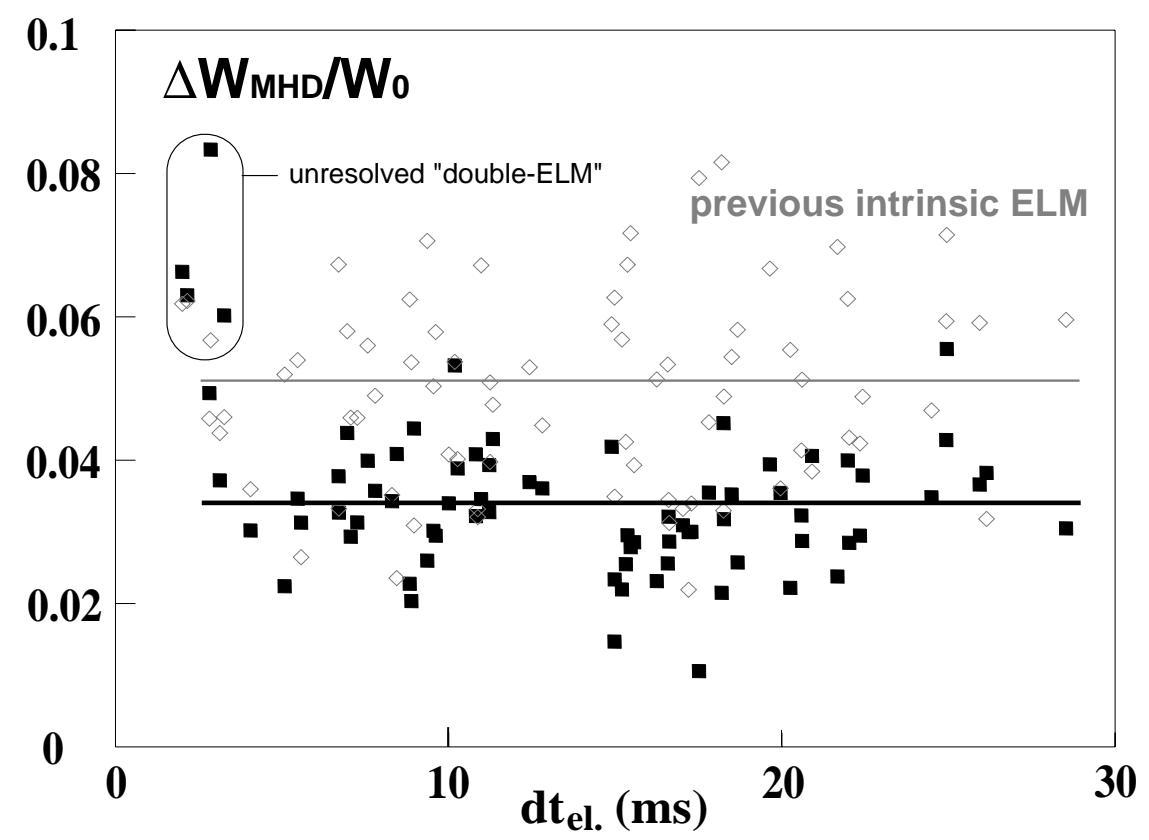

Figure 2: Normalized ELM energy loss versus time elapsed after the preceding spontaneous ELM for pelletinduced ELMs (solid black squares), and the preceding spontaneous ELMs (grey diamonds). Triggered ELMs are on average smaller in size than intrinsic ELMs (black and grey solid lines, respectively) but no indication for a correlation with $d t_{e l}$. is seen (note for small $d t_{e l}$. intrinsic and following triggered ELMs cannot be distinguished). 
The energy loss of pellet-triggered ELMs is almost uncorrelated with $d t_{e l}$. as shown in figure 2 displaying the fraction of plasma energy $\Delta W_{M H D} / W_{0}$ removed during pellet-triggered ELMs versus $d t_{e l}$. (solid squares). Here, $\Delta W_{M H D}$ is derived from the ELM induced drop in the magnetic measurement of the plasma energy, $W_{0}$ is the preELM value. Except for low $d t_{e l}$. values, where the energy loss of the triggered and previous spontaneous ELM cannot be separated any longer, $\Delta W_{M H D} / W_{0}$ remains constant, regardless of when in the ELM cycle the ELM is triggered. This is a surprising feature since the plasma energy recovers quickly after an ELM and then saturates as typical for low frequency ELMs. Unfortunately, our data did not allow to reconstruct a sufficiently precise evolution of the pedestal pressure through the ELM cycle while the pellet's fueling impact further complicates the analysis. The comparison of $\Delta W_{M H D} / W_{0}$ values found for triggered ELMs with data for spontaneous ELMs (grey diamonds in figure 2) suggests that the triggered ELMs are, on average, smaller in size than spontaneous ELMs for similar plasma background conditions. This finding seems to contradict models which relate ELM losses to the ELM frequency, or whether or not an ELM occurs shortly after a preceding ELM when the edge pressure gradient is still recovering from the ELM energy loss. Such models have been proposed by Fishpool (exponential recovery, [7]) and Herrmann [8], who suggests that the ELM loss follows the relation

$$
\frac{\Delta W_{M H D}}{W_{0}} \times f_{E L M}=\text { const }
$$

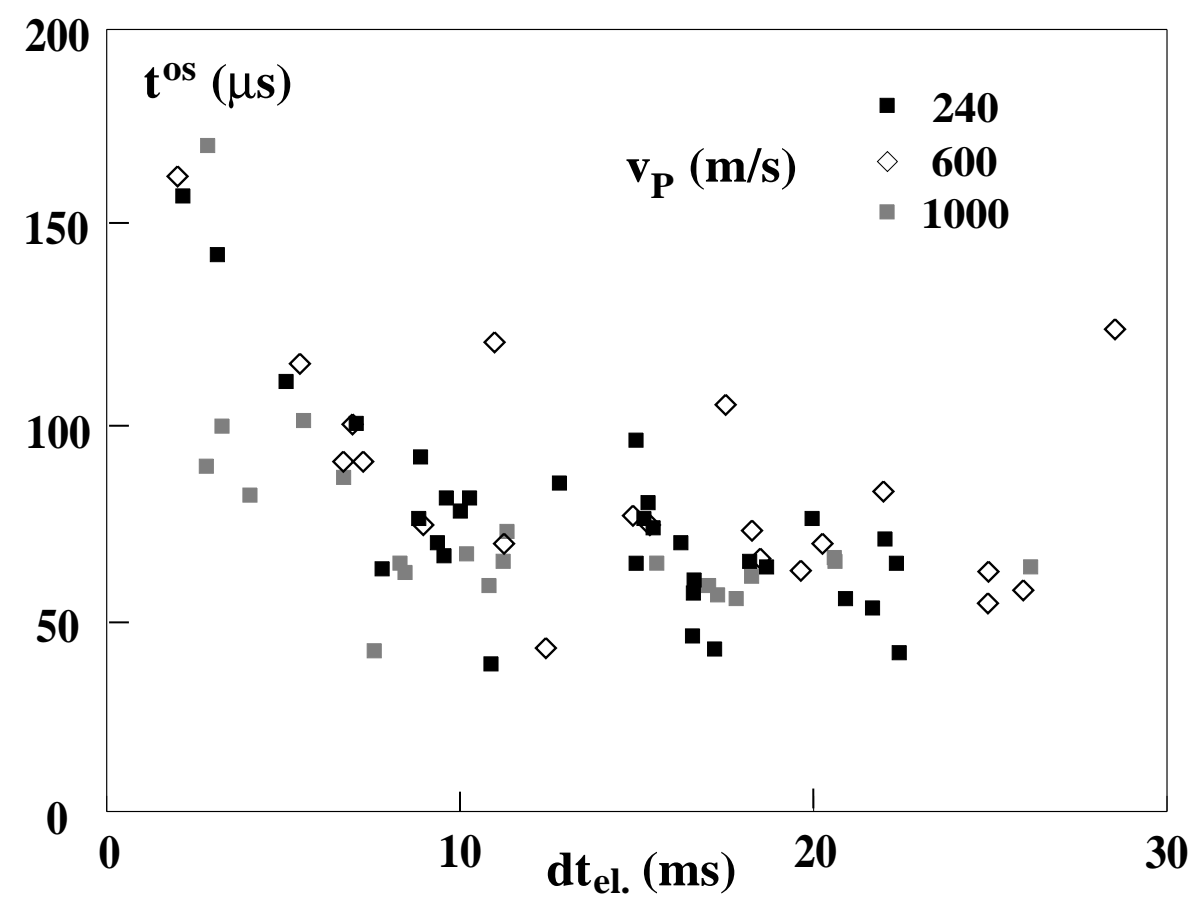

Figure 3: Onset time of triggered ELM $t^{o s}$ versus $d t_{e l}$. for different pellet velocities as indicated.

It is unclear whether such a relation between ELM frequency and ELM loss holds for the case of externally triggered ELMs and whether this is the explanation of ELM mitigation by external triggering. We address this question by studying the transient phase at the onset of a pacing sequence. In cases where a significant increase of $f_{E L M}$ was forced by imposing abruptly a sufficiently high pellet rate to establish full pacing we observed a characteristic temporal evolution of the ELM size. It appears that while the induced ELM frequency $f_{E L M}=f_{P e l}$ 
is established instantly, the reduction of the ELM size is not immediately effective. During a settling time of about $0.1-0.2 \mathrm{~s}$ ELMs at the onset of the pacing sequence gradually reduce in size from the initial intrinsic value to the final steady state value adapted to the new driving frequency. While this size adaptation is ongoing, a slight but visible reduction of the plasma energy takes place due to the still "oversized" triggered ELMs. Once the ELM size adapts to the changed frequency, steady state is reached and the plasma energy remains constant. Hence, full ELM mitigation is achieved only once this new steady state is established and ELMs triggered in the perturbation regime $f_{P e l}<f_{E L M}^{0}$ cannot be entirely identical to steady state pacing ELMs. Origin and underlying physics of the ELM size adaptation time are unclear yet, as within the accuracy of our measurements we could not detect any significant change in the density, temperature or pressure profiles in the transport barrier region.

We now examine the dynamics of the ELM onset and try to find out at which localization the seed perturbation finally causing the ELM is imposed. For this, we measured the correlation of the ELM onset delay $t^{o s}$ with respect to $d t_{e l}$. For this comparison, we use pellets of varying speed and select $t^{o s}=0$ at the time the pellets reach a reference position $4 \mathrm{~cm}$ inside the separatrix. Only for this particular position was a coincidence in the data found for the different pellet velocities as shown in figure 3. Hence, this reference point must be close to the actual position at which the pellets imposed the ELM trigger perturbation. While shortly after a previous ELM $t^{o s}$ can reach values up to $150 \mu \mathrm{s}$, is smaller at later times in the ELM cycle and asymptotically approaches $t^{o s} \approx 70 \mu \mathrm{s}$.

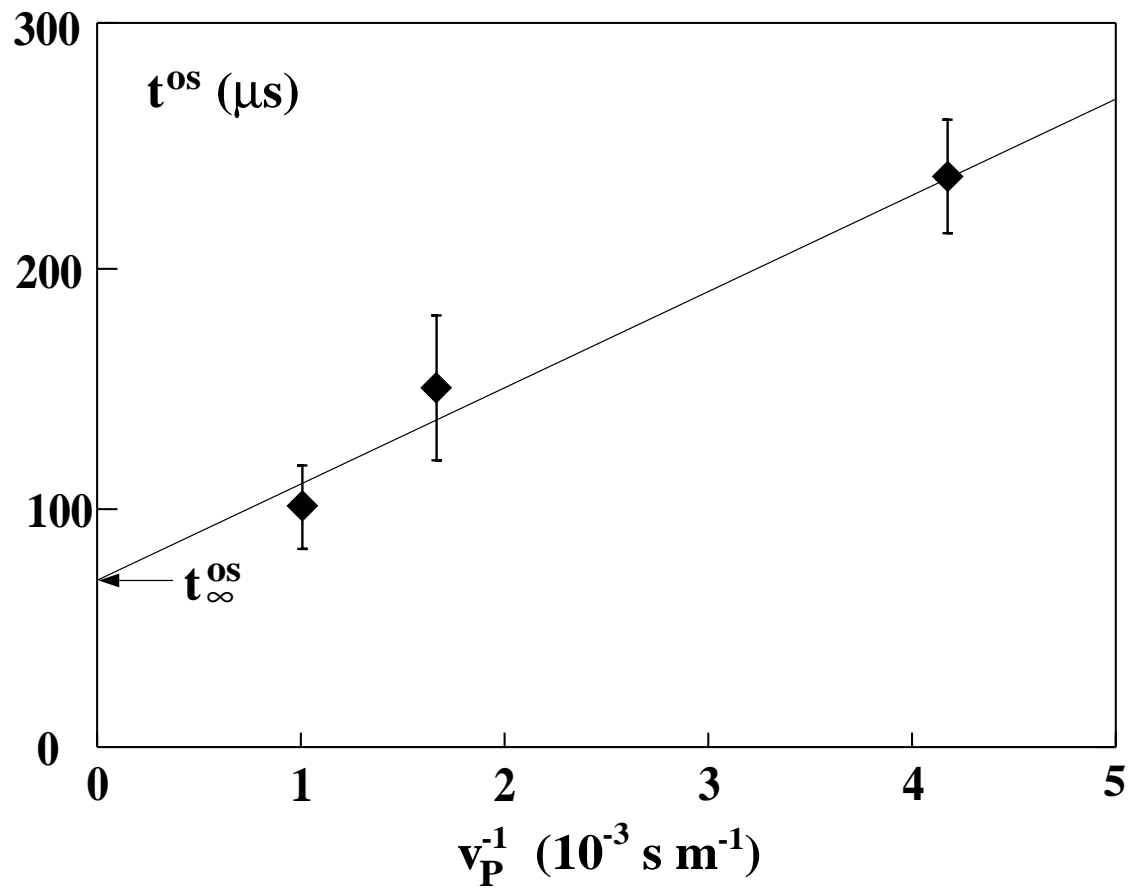

Figure 4: Onset time delay of triggered ELM versus inverse pellet velocity $v_{P}^{-1}\left(t^{o s} \equiv 0\right.$ at separatrix).

The ELM trigger delay time and the position at which a pellet triggers an ELM can be separated by a scan of the pellet velocity, which effectively varies the pellet flight time. Figure 4 shows the onset time, averaged over all pellets at a particular speed and restricted to $d t_{e l .}>10 \mathrm{~ms}$ (typically 25 pellets), as a function of reciprocal pellet velocity. In this case, the reference point is chosen such that $t^{o s} \equiv 0$ at the separatrix. The data can be described 
by an offset linear fit (solid line). The ordinate, extrapolated to infinite pellet velocity (or zero flight time), $t_{\infty}^{o s}$, represents the delay time between the passage of the pellet through the flux surface that will trigger the ELM (seed position) and the actual onset of the ELM itself. The slope indicated in the figure corresponds to the pellet path length $(40 \mathrm{~mm})$ between the reference point and the actual position where ELMs are triggered. When mapped to the low field side horizontal mid plane this distance corresponds to $13 \mathrm{~mm}$, more than half of the pedestal width $(\approx 20 \mathrm{~mm})$. This analysis implies that the ELM delay time is independent of the pellet velocity, an assumption justified by the data of Figure 3. The uncertainty of the seed position is mainly due to uncertainties in determining the pellet path itself and accuracy of mapping to the low field side.

It should be noted that the data presented here are derived by a preliminary analysis made with simplifying assumptions. For example it is assumed the pellet moving with its constant initial velocity along its designated path although observation of slightly curved pellet trajectories indicate changes of the speed. Also a simplified picture of ELMs is used which neglects its radial and poloidal structure. Moreover, a further refinement of the plasma equilibrium reconstruction is required to fully resolve the impact of the ELMs. A more sophisticated analysis taking into account all these effects is in progress [6].

\section{Discussion and conclusions}

Comparison of the pellet and supersonic gas jet injections shows that for ELM triggering a perturbation is required in the transport barrier region and significantly inside the separatrix. Pellets can easily reach this region and even smallest pellets are found to trigger ELMs, while the gas jet does not lead to immediate triggering of ELMs. The smallest and fastest pellets deposit about $10^{21}-10^{22} \mathrm{D} / \mathrm{m}^{3}$. The largest gas jet events (for detailed profiles see [4]) goes up to $10^{25}-10^{26} \mathrm{D} / \mathrm{m}^{3}$, orders of magnitude larger than with pellets, but located outside the separatrix. We conclude that the sensitivity for ELM trigger perturbations is highly localized in the steep gradient region of the $\mathrm{H}$-mode edge. Of course, it should be noted that the perturbation pattern imposed by pellet and gas jet are rather different. While the pellet imposes a quite compact initial perturbation stemming from the ablation plasmoid, the gas jet is less localized. Obviously these differences in the perturbation pattern are enclosing the required trigger threshold. It should be also mentioned that different injection locations (HFS for pellets, LFS for gas jet) do not play a significant role since ELM triggering was also reported for pellet launch from the LFS [9].

The region of the maximum density change due to the gas jet, and the region in which pellets trigger ELMs (according to the analysis above) are depicted in Figure 5. The width of the latter region represents the estimated radial uncertainty due to our analysis. For comparison, a typical H-mode edge electron pressure profile is shown as a function of radius at the outer horizontal mid plane. Obviously ELMs are triggered when the perturbation reaches the middle of the steep gradient region, where the strongest pressure gradient drive for the ELM instability exists. If the observations are interpreted as a radial variation of a critical density perturbation needed to trigger ELMs, then the perturbation strength varies by at least a factor of 100 between the steep gradient region and the scrape-off layer.

In a scan of pellet velocity, we can separate the pellet flight time from the delay time between the pellet passing the critical region and the first observation of ELM growth. Independently of pellet parameters, and for the entire ELM cycle with the exception of the first 5-10 ms after an ELM crash, this time amounts to $t_{\infty}^{o s} \sim 70 \mu \mathrm{s}$. Right 


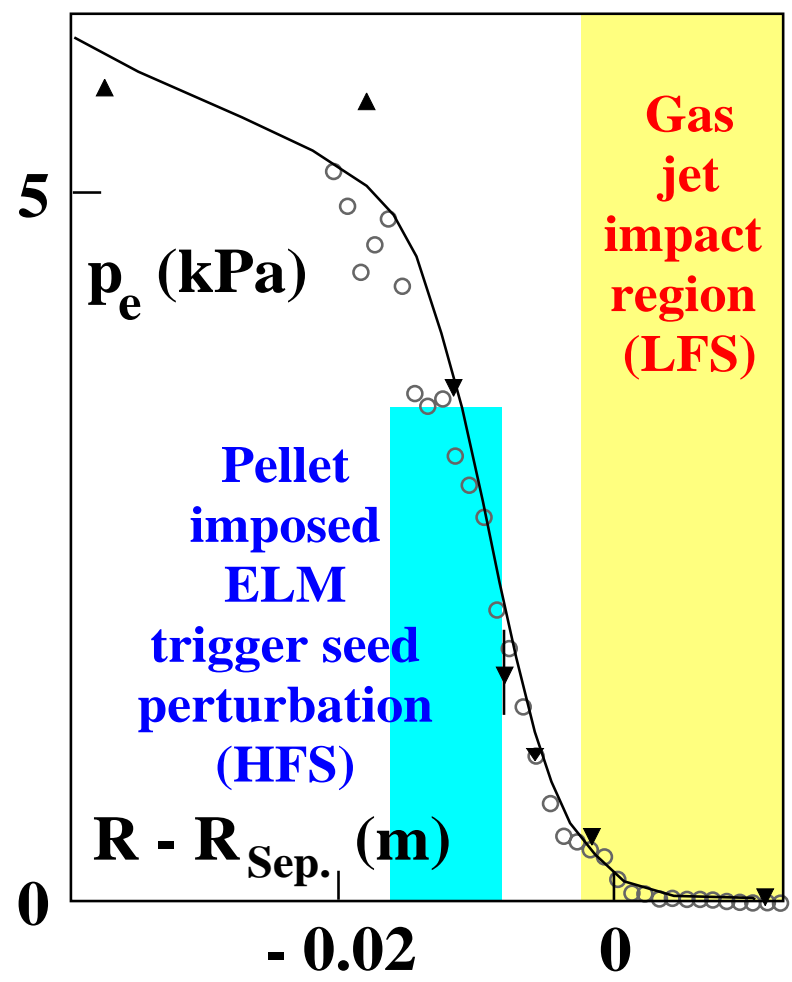

Figure 5: Radial extent of the region where pellets induce ELMs compared with the gas jet deposition region vs. radial distance to the separatrix at outer mid plane. Overlaid is a typical electron pressure profile (triangles: Thomson scattering, circles: Li beam, solid line: tanh-fit to the data).

after an ELM, this time can be longer.

At present, no complete picture of the ELM trigger mechanism exists. Our value of $t_{\infty}^{o s}$ sets an upper bound to the ELM growth time which rules out slower processes. Conceptually, one may split up this time interval into a time needed for the pellet mass to distribute sufficiently in poloidal direction to trigger an ELM and the actual growth time of the ELM instability. If we assume the ELM trigger has to take place at the low field side (in the region of unfavorable field line curvature), then the pellet-induced perturbation might be communicated with ionic sound speed expansion of the pellet cloud (time delay of the order of $50 \mu \mathrm{s}$ ) or Alfvén velocity $(\sim 5 \mu \mathrm{s})$. However, an identification of a transport mechanism is not yet possible. This question is addressed by experiments (in preparation) which involve pellet injection from different poloidal locations.

We find that the ELM energy loss is essentially independent from the time $d t_{e l}$. after the preceding ELM event. This observation seems to be, at first sight, in contradiction with models relating ELM losses to the ELM frequency and also ELM mitigation by pacing itself. However, the energy loss of isolated triggered ELMs (i.e. ELMs in a plasma dominated by spontaneous ELMs) is different from that of the spontaneous ELMs, and it is also different from the energy loss in a quasi-stationary fast pacing sequence where each ELM is pellet-triggered. In this last case, and after an initial settling time interval of about $100 \mathrm{~ms}$, the ELM energy loss can be described by the relation $\frac{\Delta W_{M H D}}{W_{0}} \times f_{E L M}=$ const., where $f_{E L M}$ is now the average ELM frequency generated by the pellet pacing. To gain better insight more information on the pedestal evolution through a perturbed ELM cycle is required as well as the 
radial extend of the triggered ELM. For the present study, large pellets having more mass than needed to trigger ELM are used, whereas these masses hamper the analysis. Also, the pellets applied so far penetrate much deeper than needed to trigger ELMs. Thus, there is still significant headroom for improving ELM pacing by developing injector systems that are capable to launch smaller pellets with reduced fueling. As well, such systems could enable to apply more flexibility in the imposed perturbation pattern helping to investigate ELM trigger conditions at enhanced spatial and temporal resolution. Even better prospects exists for larger machines, which have larger particle inventory and lower ELM frequency than current mid-size tokamaks such as ASDEX Upgrade. Here, a sufficient local perturbation can be expected from pellets of negligible global impact. Furthermore, smaller

pellet injection rates are sufficient to fully control the ELM events. In conclusion, pellet injection can provide a technically simple and straight-forward technique for ELM pacing and ELM loss mitigation in large tokamaks.

\section{References}

[1] LANG, P. T. et al., Nucl. Fusion 43 (2003) 1110.

[2] DEGELING, A. et al., Plasma Phys. Controlled Fusion 45 (2003) 1637.

[3] LANG, P. T. et al., Nucl. Fusion 44 (2004) 665.

[4] LANG, P. T. et al., Plasma Phys. Controlled Fusion 47 (2005) 1495.

[5] MC NEILL, D., J. Nucl. Mater. 162-164 (1989) 476.

[6] KOCSIS, G. et al., in preparation for submission to Plasma Phys. Controlled Fusion .

[7] FISHPOOL, G. M., Nucl. Fusion 38 (1998) 1373.

[8] HERRMANN, A., Plasma Phys. Controlled Fusion 44 (2002) 883.

[9] LANG, P. T. et al., Nucl. Fusion 36 (1996) 1531, Corrigendum in Nucl. Fus. 36 (1996) 153. 